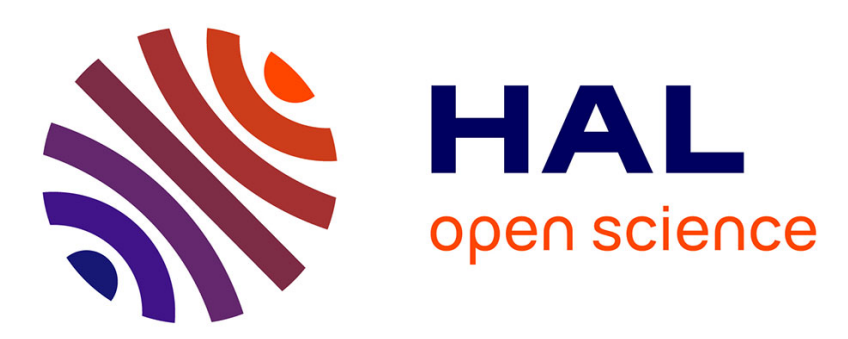

\title{
Director Field Analysis to Explore Local White Matter Geometric Structure in diffusion MRI
}

Jian Cheng, Peter J. Basser

\section{To cite this version:}

Jian Cheng, Peter J. Basser. Director Field Analysis to Explore Local White Matter Geometric Structure in diffusion MRI. 25th Biennal International Conference on Information Processing in Medical Imaging (IPMI'17), Jun 2017, Boone, United States. hal-01511573

\section{HAL Id: hal-01511573 \\ https://hal.science/hal-01511573}

Submitted on 21 Apr 2017

HAL is a multi-disciplinary open access archive for the deposit and dissemination of scientific research documents, whether they are published or not. The documents may come from teaching and research institutions in France or abroad, or from public or private research centers.
L'archive ouverte pluridisciplinaire HAL, est destinée au dépôt et à la diffusion de documents scientifiques de niveau recherche, publiés ou non, émanant des établissements d'enseignement et de recherche français ou étrangers, des laboratoires publics ou privés. 


\title{
Director Field Analysis to Explore Local White Matter Geometric Structure in diffusion MRI
}

\author{
Jian Cheng, Peter J. Basser \\ SQITS, NIBIB, NICHD, National Institutes of Health \\ jian.cheng@nih.gov, pjbasser@helix.nih.gov
}

\begin{abstract}
In diffusion MRI, a tensor field or a spherical function field, e.g., an Orientation Distribution Function (ODF) field, are estimated from measured diffusion weighted images. In this paper, inspired by microscopic theoretical treatment of phases in liquid crystals, we introduce a novel mathematical framework, called Director Field Analysis (DFA), to study local geometric structural information of white matter from the estimated tensor field or spherical function field. 1) We propose Orientational Order (OO) and Orientational Dispersion (OD) indices to describe the degree of alignment and dispersion of a spherical function in each voxel; 2) We estimate a local orthogonal coordinate frame in each voxel with anisotropic diffusion; 3) Finally, we define three indices to describe three types of orientational distortion (splay, bend, and twist) in a local spatial neighborhood, and a total distortion index to describe distortions of all three types. To our knowledge, this is the first work to quantitatively describe orientational distortion (splay, bend, and twist) in diffusion MRI. The proposed scalar indices are useful to detect local geometric changes of white matter for voxelbased or tract-based analysis in both DTI and HARDI acquisitions.
\end{abstract}

\section{Introduction}

Diffusion MRI is a unique non-invasive imaging technique to explore white matter in human brain. Diffusion Tensor Imaging (DTI) [3] is used to reconstruct a tensor field from diffusion weighted images (DWIs). High Angular Resolution Diffusion Imaging [13], without the assumption of Gaussian diffusion, is used to reconstruct a general function field from DWIs, e.g., an Orientation Distribution Function (ODF) or Ensemble Average Propagator (EAP) field. Exploring meaningful information from the reconstructed tensor field or spherical function field is of interest in many application areas, which makes diffusion MRI a powerful means to study white matter.

Some scalar indices have been proposed to be estimated voxel-wise from tensors/ODFs/EAPs. For DTI, the mean diffusivity and Fractional Anisotropy (FA) are widely used biologically meaningful descriptors [8]. For HARDI, the generalized FA [13], Orientation Dispersion (OD) [14], etc., were proposed for ODFs and EAPs. Some existing works have extracted local geometric information by considering spatial gradients of tensor field. [7] 
demonstrated that the norm of the spatial gradient of the tensor field can be useful for detecting boundaries between white matter, CSF and grey matter. [5] proposed tangents of scalar invariants and rotation tangents which are 2nd order tensors, and also proposed projecting the 3rd order spatial gradient tensor onto these 2nd order tangents to obtain the spatial direction with the largest change of scalar indices or rotation of tensors. Based on the rotation tangents of tensors, [10] proposed fiber curving and fiber dispersion indices. These studies are all based on spatial gradients of tensor fields from DTI. However, to our knowledge, there is no work making connections between general spherical function fields from HARDI data and local geometric structure (splay, bend and twist) of white matter.

Torsion and curvature of a fiber tract [2] were proposed based on the Frenet frame along the tract. [12] used Frenet frame as a prior to regularize the data and estimate ODFs in voxels. These works on the Frenet frame studied geometric information along a single tract. However, tractography is known to be sensitive to a large number of parameters, and any flaws in the reconstructed tracts due to noise or parameter selection will inevitably be reflected in the subsequently extracted geometric information. [9] proposed moving frames determined by the geometry of cardiac data, and calculated Maurer-Cartan connections. However this method cannot work for general diffusion MRI data, and did not consider the sign ambiguity in the frame.

Orientational order parameter is well-established to describe the degree of alignment in liquid crystals [1]. [11] calculated the order parameter map by estimating variance of microscopic diffusion parameters from the contrast between signals measured by directional and isotropic diffusion encoding. However, it cannot be used for general DTI and HARDI data.

In this paper, inspired by theoretical analysis of orientation and distortion for liquid crystals, we propose a unified framework, called Director Field Analysis (DFA), to study local geometric information of white matter. DFA works for both tensor field from DTI and spherical function field from HARDI. At the voxel level, 1) the Orientational Order index (OO) is defined for the spherical function in voxel with a given axis, e.g., the ODF with the principal direction, and the Orientational Dispersion index (OD) is defined as 1-OO. 2) The principal direction is extracted from the spherical function in a anisotropic voxel. In a local neighborhood level, 1) a local orthogonal frame is defined for each anisotropic voxel with the first axis as the extracted principal direction; 2) three distortion indices (splay, bend, twist) and a total distortion index are defined based on spatial directional derivatives of the principal direction.

\section{Method: Director Field Analysis}

\subsection{Orientational Order and Dispersion}

The NODDI model is increasingly used to study neurite orientation dispersion [14]. NODDI uses the Watson distribution in Eq. (1) to model the 
orientation distribution function with a single orientation,

$$
f(\mathbf{u} \mid \boldsymbol{n})=\frac{1}{4 \pi M(1 / 2,3 / 2, \kappa)} \exp \left(\kappa\left(\mathbf{u}^{T} \boldsymbol{n}\right)^{2}\right), \quad \mathbf{u} \in \mathbb{S}^{2}
$$

where $M$ is the confluent hypergeometric function, $\boldsymbol{n} \in \mathbb{S}^{2}$ is a given axis. Note that the original formula in [14], which has no unit integral in $\mathbb{S}^{2}$, missed $4 \pi$. An orientation dispersion index (OD) was defined as $\mathrm{OD}_{w}=$ $\frac{2}{\pi} \arctan \left(\frac{1}{\kappa}\right)$, where we denote it as $\mathrm{OD}_{w}$ because it can not be used for ODFs with general shapes, with more than one peak.

For a general spherical function $f(\mathbf{u}), \mathbf{u} \in \mathbb{S}^{2}$, we propose the orientational order index $(O O)$ from the theory of liquid crystals [1] to describe the orientation or dispersion of a general spherical function along a given axis $\boldsymbol{n}$ :

$$
\mathrm{OO}(\boldsymbol{n})=\int_{\mathbf{u} \in \mathbb{S}^{2}} P_{2}\left(\mathbf{u}^{T} \boldsymbol{n}\right) f(\mathbf{u}) \mathrm{d} S=\int_{\mathbb{S}^{2}} \frac{3\left(\mathbf{u}^{T} \boldsymbol{n}\right)^{2}-1}{2} f(\mathbf{u}) \mathrm{d} S
$$

where $P_{2}$ is the second-order Legendre polynomial. By definition Eq. (2) is an integral transform in $\mathbb{S}^{2}$ which converts $f(\mathbf{u})$ to another spherical function $\mathrm{OO}(\boldsymbol{n})$, and the kernel is $P_{2}\left(\mathbf{u}^{T} \boldsymbol{n}\right)$, similar to the Funk-Radon transform used in Q-Ball imaging [13], where the kernel is $\delta\left(\mathbf{u}^{T} \boldsymbol{n}\right)$. We call Eq. (2) the Orientational Order Transform (OOT). Although $\mathrm{OO}(\boldsymbol{n})$ is a spherical function, it is a scalar index when $\boldsymbol{n}$ is chosen as a physically meaningful axis, e.g., $f(\mathbf{u})$ takes its maximal value at $\boldsymbol{n}$. Let $\theta$ be the angle between $\mathbf{u}$ and axis $\boldsymbol{n}$, then $P_{2}\left(\mathbf{u}^{T} \boldsymbol{n}\right)=\frac{3 \cos ^{2} \theta-1}{2}$. Thus if $f(\mathbf{u})$ is a Probability Density Function (PDF) in sphere, then $\mathrm{OO}$ is $\left\langle\frac{3 \cos ^{2} \theta-1}{2}\right\rangle$, where $\langle\cdot\rangle$ signifies the expectation operation. $\left\langle\cos ^{2} \theta\right\rangle$ is the expectation of squared projected length of $\mathbf{u}$ onto the axis $\boldsymbol{n}$. By definition, when $f(\mathbf{u})$ is a PDF, then we have $\mathrm{OO} \in[-0.5,1]$. If $f(\mathbf{u})=\delta\left(\mathbf{u}^{T} \boldsymbol{n}-1\right)$, i.e. the delta function along $\boldsymbol{n}$ axis, then $\mathrm{OO}=1$. If $f(\mathbf{u})=0, \forall \mathbf{u} \in \mathbb{S}^{2}$ such that $\mathbf{u}^{T} \boldsymbol{n} \neq 0$, then $\mathrm{OO}=-0.5$. If $f(\mathbf{u})$ is the isotropic PDF, i.e. $f(\mathbf{u})=\frac{1}{4 \pi}$, then $\mathrm{OO}=0$. In practice, if we choose the axis $\boldsymbol{n}$ such that $f(\mathbf{u})$ takes its global maximal value, then $\mathrm{OO}$ is normally non-negative. We define the orientational dispersion, $\mathrm{OD}=1-\mathrm{OO}$. Then $\mathrm{OD} \in[0,1.5]$.

Axisymmetric spherical functions. When $f(\mathbf{u})$ is axisymmetric, and its axis is given by $\boldsymbol{n}_{0}$, i.e., $f(\mathbf{u})=f^{\prime}\left(\mathbf{u}^{T} \boldsymbol{n}_{0}\right)$, where $f^{\prime}(x)$ is the corresponding scalar function defined in $[-1,1]$, then OOT has a closed form:

$$
\begin{aligned}
\mathrm{OO}(\boldsymbol{n}) & =\int_{0}^{\pi}\left(\int_{0}^{2 \pi} P_{2}(\cos \theta \cos t+\sin \theta \cos t \sin \phi) \mathrm{d} t\right) f^{\prime}(\cos \theta) \mathrm{d} \theta \\
& =\frac{(1+3 \cos (2 \phi)) \pi}{2} a_{2}=\frac{1+3 \cos (2 \phi)}{4} \mathrm{OO}\left(\boldsymbol{n}_{0}\right)
\end{aligned}
$$

where $\phi=\arccos \left(\left|\boldsymbol{n}^{T} \boldsymbol{n}_{0}\right|\right)$ is the angle between $\boldsymbol{n}$ and the axis $\boldsymbol{n}_{0}$, and $a_{2}=$ $\int_{-1}^{1} P_{2}(x) f^{\prime}(x) \mathrm{d} x$ is the second-order Legendre coefficient of $f^{\prime}(x)$. Note that if $a_{2}>0$, when $\boldsymbol{n}=\boldsymbol{n}_{0}, \phi=0$, then $\mathrm{OO}=2 \pi a_{2}$ is the global maximum of $\mathrm{OO}(\boldsymbol{n})$.

\footnotetext{
${ }^{1}$ https://en.wikipedia.org/wiki/Liquid_crystal
} 

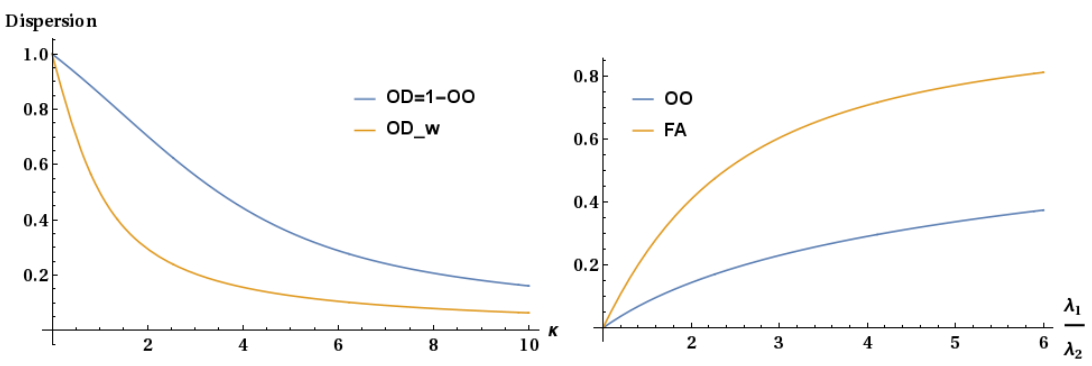

Fig. 1. Left: dispersion indices of a Watson distribution as functions of $\kappa$. Right: $\mathrm{OO}$ and FA of prolate tensors $\left(\lambda_{2}=\lambda_{3}\right)$ as functions of $\frac{\lambda_{1}}{\lambda_{2}}$.

In the following paper, without any ambiguity, we will use OO to denote $\mathrm{OO}\left(\boldsymbol{n}_{0}\right)$ for axisymmetric spherical functions.

Watson distributions. The Watson distribution defined in Eq. (1) is axisymmetric with the axis $\boldsymbol{n}_{0}$. Thus, based on the above analysis of axisymmetric spherical functions, we have $\mathrm{OO}(\boldsymbol{n})=\frac{1+3 \cos (2 \phi)}{4} \mathrm{OO}$, and

$$
\mathrm{OO}=\frac{3 e^{\kappa}}{2 \sqrt{\kappa \pi} \operatorname{Erfi}(\sqrt{\kappa})}-\frac{3+2 \kappa}{4 \kappa}
$$

where $\operatorname{Erfi}(x)=\frac{2}{\sqrt{\pi}} \int_{0}^{x} \exp \left(t^{2}\right) \mathrm{d} t$ is the imaginary error function. Then OD $=$ $1-\mathrm{OO}$. The left part of Fig. 1 shows the above OD and $\mathrm{OD}_{w}$ as functions of $\kappa$, where the axis $\boldsymbol{n}$ is set as the Watson distribution's axis. Both dispersion indices decrease as $\kappa$ increases. Based on the derivation of $\kappa, \mathrm{OD}_{w}$ is more sensitive for changes of $\kappa$ when $\kappa$ is small $(<2)$, while it is less sensitive when $\kappa$ is large $(>2)$. Compared with $\mathrm{OD}_{w}$, the change of $\mathrm{OD}$ is smoother for the change of $\kappa$ over the entire range of $\kappa$.

Tensors. For a tensor D in DTI, OOT is defined for its ODF, i.e.,

$$
\Phi(\mathbf{u} \mid \mathbf{D})=\frac{1}{4 \pi|\mathbf{D}|^{\frac{1}{2}}} \frac{1}{\left(\mathbf{u}^{T} \mathbf{D}^{-1} \mathbf{u}\right)^{\frac{3}{2}}} .
$$

which is a PDF on the unit sphere. When the three eigenvalues of $\mathbf{D}$ satisfies $\lambda_{1}>\lambda_{2}=\lambda_{3}>0, \Phi(\mathbf{u} \mid \mathbf{D})$ is axisymmetric with the axis $\boldsymbol{v}_{1}$ that is the principal eigenvector of D. OOT has a closed-form expression in Eq. (3), and

$$
\mathrm{OO}=\frac{\sqrt{\lambda_{1}-\lambda_{2}}\left(2 \lambda_{1}+\lambda_{2}\right)-3 \lambda_{1} \sqrt{\lambda_{2}} \arctan \left(\sqrt{\frac{\lambda_{1}-\lambda_{2}}{\lambda_{2}}}\right)}{2\left(\lambda_{1}-\lambda_{2}\right)^{\frac{3}{2}}} .
$$

The right panel of Fig. 1 shows OO and FA as functions of $\lambda_{1} / \lambda_{2}$, where we set $\boldsymbol{n}=\boldsymbol{v}_{1}$. Both OO and FA increases as $\lambda_{1} / \lambda_{2}$ increases. Thus OO can be seen as a type of anisotropy index for tensors. For general tensors with $\lambda_{1}>\lambda_{2}>\lambda_{3}$, no such closed form solution like Eq. (6) and Eq. (3) exist, but we can calculate $\mathrm{OO}$ using the spherical harmonic representation of the ODF.

Spherical harmonic representation. For a general spherical function $f(\mathbf{u}), \mathrm{OO}$ and $\mathrm{OD}$ can be analytically calculated from its rotated spherical 

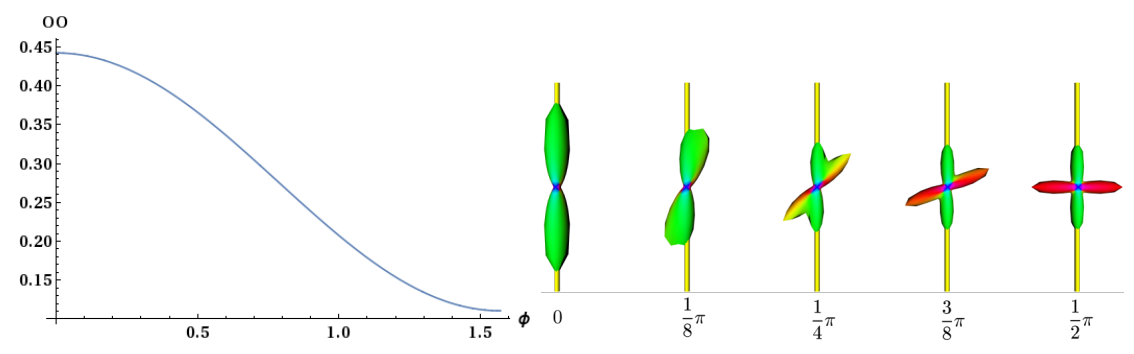

Fig. 2. OO for mixture tensor model. Left: $O O$ as a function of the angle between two tensor components. Right: ODF glyphs for different crossing angles, where the yellow tube shows the $y$-axis to calculate OO.

harmonic coefficients. Considering $f(\mathbf{u})$ is a real function in sphere, it can always be linearly represented by the real Spherical Harmonic (SH) basis $\left\{Y_{l}^{m}(\mathbf{u})\right\}$, i.e., $f(\mathbf{u})=\sum_{l, m} c_{l}^{m} Y_{l}^{m}(\mathbf{u})$, where $Y_{l}^{m}(\mathbf{u})$ is the real Spherical Harmonic (SH) basis. For any rotation matrix, the $\mathrm{SH}$ coefficients of the rotated function can be calculated with very high accuracy based on Wigner D-matrix 2 , or based on fitting rotated function samples [6]. Let $\mathbf{R}$ be the rotation matrix which rotates the axis $\boldsymbol{n}$ to $\boldsymbol{z}$-axis, and $\left\{a_{l}^{m}\right\}$ be the real SH coefficients of the rotated function $(R f)(\mathbf{u})=f\left(\mathbf{R}^{-1} \mathbf{u}\right)$, considering the orthogonality of real SH basis and $Y_{2}^{0}(\theta, \phi)=\sqrt{\frac{5}{4 \pi}} P_{2}(\cos \theta)$, we have

$$
\mathrm{OO}(\boldsymbol{n})=\int_{\mathbf{u} \in \mathbb{S}^{2}} P_{2}\left(\mathbf{u}^{T} \boldsymbol{n}\right) f(\mathbf{u}) \mathrm{d} S=\int_{\mathbf{u} \in \mathbb{S}^{2}} P_{2}(\cos \theta) \sum_{l, m} a_{l}^{m} Y_{l}^{m}(\theta, \phi) \mathrm{d} S=\sqrt{\frac{4 \pi}{5}} a_{2}^{0}
$$

Note that $\mathrm{OO}(\boldsymbol{n})$ is only determined by the rotated $\mathrm{SH}$ coefficient $a_{2}^{0}$ which is only related to $\left\{c_{2}^{m}\right\}_{-2 \leq m \leq 2}$ and the axis $\boldsymbol{n}$, based on the property of the $\mathrm{SH}$ basis. Thus $\mathrm{OO}(\boldsymbol{n})$ is only related to the $\mathrm{SH}$ coefficients of $f(\mathbf{u})$ with $l=2$.

Mixture model. OOT in Eq. (2) is a linear transform. Thus if $f(\mathbf{u})=$ $\sum_{i} w_{i} f_{i}(\mathbf{u})$ is the ODF of a mixture of models, where $f_{i}(\mathbf{u})$ is the ODF for the $i$ th model, and $w_{i}$ is the weight, then $\mathrm{OO}(\boldsymbol{n})=\sum_{i} w_{i} \mathrm{OO}_{i}(\boldsymbol{n})$ is a mixture of $\mathrm{OO}$ functions. Fig. 2 illustrates $\mathrm{OO}$ for a two-tensor model with a crossing angle $\phi$, where two tensors share the same eigenvalues $[1.7,0.2,0.2] \times 10^{-3} \mathrm{~mm}^{2} / \mathrm{s}$, the weights are 0.5 and 0.5 , and one tensor component is along $y$-axis and the other one rotates from $y$-axis to $x$-axis. Based on Eq. (3) and Eq. (6), OO for the mixture model can be analytically calculated.

\subsection{Director and Local Orthogonal Frame}

In a voxel with anisotropic diffusion, the orientations where the anisotropic ODF takes its local peak (i.e., local maximal values) are normally considered as local fiber directions in that voxel. A normal peak detection algorithm for

\footnotetext{
${ }^{2}$ https://en.wikipedia.org/wiki/Spherical_harmonics
} 


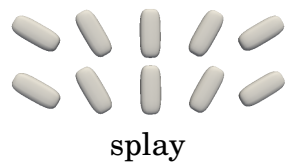

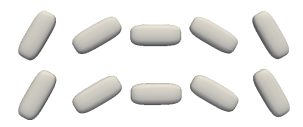

bend

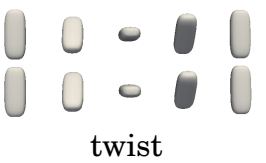

twist

Fig. 3. Demonstration of three types of distortions.

ODFs performs a grid search in a spherical mesh, then refines the solution using a gradient ascent on the continuous sphere. In order to avoid including small peaks produced by noise, only peaks whose value larger than a threshold percentage (e.g., 0.5) of the largest ODF value are counted.

We define a director as a vector $\boldsymbol{v}$ which is equivalent to its negative $-\boldsymbol{v}$. Normally ODFs are antipodally symmetric, thus a peak is a director. After peak detection, for each voxel $\boldsymbol{x}$, we obtain a discrete spherical function $g(\mathbf{u}, \boldsymbol{x})=\sum_{i} f\left(\mathbf{u}_{i}, \boldsymbol{x}\right) \boldsymbol{\delta}\left(\mathbf{u}-\mathbf{u}_{i}\right)$ from the continuous spherical function $f(\mathbf{u}, \boldsymbol{x})$, where $\left\{\mathbf{u}_{i}\right\}$ are local peaks. This discrete spherical function field is called a director field, or peak field. We propose extracting a local orthogonal frame in each anisotropic voxel from the detected peak field. The orthogonal frame has three orthogonal orientations. The first orientation is the principal direction where the ODF takes its global maximum $\mathbf{u}_{1}$, i.e. $f\left(\mathbf{u}_{1}, \boldsymbol{x}\right)>f\left(\mathbf{u}_{i}, \boldsymbol{x}\right)$, $\forall i \neq 1$. We call it the principal director of the anisotropic voxel. The other two orientations are in the orthogonal plane of the principal direction. Considering $f(\mathbf{u})$ is normally antipodally symmetric in diffusion MRI, all these orientations are equivalent with their antipodal ones. Thus, we define a weighted sum of dyadic tensors in voxel $x$ :

$$
T_{x}=\sum_{\boldsymbol{y} \in \Omega_{\boldsymbol{x}}} \sum_{i} w(\boldsymbol{y}, \boldsymbol{x}) f\left(\mathbf{u}_{i}, \boldsymbol{y}\right)\left(\mathbf{u}_{i}-\left(\mathbf{u}_{i}^{T} \mathbf{u}_{1}\right) \mathbf{u}_{1}\right)\left(\mathbf{u}_{i}-\left(\mathbf{u}_{i}^{T} \mathbf{u}_{1}\right) \mathbf{u}_{1}\right)^{T}
$$

where $\Omega_{x}$ is a local neighborhood of voxel $\boldsymbol{x}, w(\boldsymbol{y}, \boldsymbol{x})$ is the spatial weight which is normally set to be proportional to $\exp \left(-\frac{\|\boldsymbol{y}-\boldsymbol{x}\|^{2}}{2 \sigma^{2}}\right), \delta$ which is normally set as 1 voxel controls spatial weight concentration, $\left(\mathbf{u}_{i}-\left(\mathbf{u}_{i}^{T} \mathbf{u}_{1}\right) \mathbf{u}_{1}\right)\left(\mathbf{u}_{i}-\right.$ $\left.\left(\mathbf{u}_{i}^{T} \mathbf{u}_{1}\right) \mathbf{u}_{1}\right)^{T}$ is the outer product of the projected orientation $\mathbf{u}_{i}$ onto the orthogonal plane of $\mathbf{u}_{1} . T_{x}$ has at most two non-zero eigenvalues, because it is defined in the orthogonal plane. The eigenvector for the largest absolute eigenvalue is set as the second orientation of the orthogonal frame. The third orientation in the orthogonal frame is set as the cross product of the first two orientations. Note that the proposed local orthogonal frame determined by the local change of principal director, is different from the moving frame in [9], which is determined by heart geometry.

\subsection{Local Distortion Indices: Splay, Bend, and Twist}

There are three types of distortions 1 for the director field as demonstrated in Fig. 3. 1) splay: bending occurs perpendicular to the director. 2) bend: the distortion is parallel to the director and molecular axis. 3) twist: neighboring 
directors are rotated with respect to one another, rather than aligned. These three fundamental distortions can describe complex geometric patterns.

With the local orthogonal frame $\left\{\mathbf{u}_{1}(\boldsymbol{x}), \mathbf{u}_{2}(\boldsymbol{x}), \mathbf{u}_{3}(\boldsymbol{x})\right\}$ obtained at each anisotropic voxel $\boldsymbol{x}$, we propose three scalar indices to describe the three types of local orientational distortions of white matter, and a total distortion index as:

$$
\begin{aligned}
& \text { Splay index: } s=\sqrt{\left(\mathbf{u}_{2}^{T} \frac{\partial \mathbf{u}_{1}}{\partial \mathbf{u}_{2}}\right)^{2}+\left(\mathbf{u}_{3}^{T} \frac{\partial \mathbf{u}_{1}}{\partial \mathbf{u}_{3}}\right)^{2}} \\
& \text { Bend index: } b=\sqrt{\left(\mathbf{u}_{2}^{T} \frac{\partial \mathbf{u}_{1}}{\partial \mathbf{u}_{1}}\right)^{2}+\left(\mathbf{u}_{3}^{T} \frac{\partial \mathbf{u}_{1}}{\partial \mathbf{u}_{1}}\right)^{2}} \\
& \text { Twist index: } \quad t=\sqrt{\left(\mathbf{u}_{2}^{T} \frac{\partial \mathbf{u}_{1}}{\partial \mathbf{u}_{3}}\right)^{2}+\left(\mathbf{u}_{3}^{T} \frac{\partial \mathbf{u}_{1}}{\partial \mathbf{u}_{2}}\right)^{2}} \\
& \text { Total distortion index: } \quad d=\sqrt{s^{2}+b^{2}+t^{2}}
\end{aligned}
$$

where $\frac{\partial \mathbf{u}_{1}}{\partial \mathbf{u}_{i}}, i=1,2,3$, is the spatial directional derivative of director field $\mathbf{u}_{1}(\boldsymbol{x})$ respectively along $\mathbf{u}_{i}$, i.e.,

$$
\frac{\partial \mathbf{u}_{1}}{\partial \mathbf{u}_{i}}=\lim _{k \rightarrow 0} \frac{\mathbf{u}_{1}\left(\boldsymbol{x}+k \mathbf{u}_{i}\right)-\mathbf{u}_{1}\left(\boldsymbol{x}-k \mathbf{u}_{i}\right)}{2 k}
$$

We define these indices using squared value of $\mathbf{u}_{j}^{T} \frac{\partial \mathbf{u}_{1}}{\partial \mathbf{u}_{i}}$ to avoid the sign ambiguity of $\left\{\mathbf{u}_{i}\right\}$ and $\left\{\frac{\partial \mathbf{u}_{1}}{\partial \mathbf{u}_{i}}\right\}$.

It is challenging to numerically calculate the three spatial directional derivatives $\left\{\frac{\partial \mathbf{u}_{1}}{\partial \mathbf{u}_{i}}\right\}$, because the extracted local coordinate frame $\left\{\mathbf{u}_{i}(\boldsymbol{x})\right\}$ is ambiguous with respect to its sign. In other words, $\mathbf{u}_{i}$ is equivalent to $-\mathbf{u}_{i}$. We propose calculating the above spatial directional derivatives using rotation matrices. See Algorithm 1 . The algorithm first calculates three rotation matrices respectively along $x, y, z$ axes, which is analogous to the spatial gradient of a vector field. Then $\frac{\partial \mathbf{u}_{1}}{\partial \mathbf{u}_{i}}$ is numerically approximated by $\mathbf{u}_{1}\left(\boldsymbol{x}+\mathbf{u}_{i}\right)-\mathbf{u}_{1}\left(\boldsymbol{x}-\mathbf{u}_{i}\right)$, where $\mathbf{u}_{1}\left(\boldsymbol{x}+\mathbf{u}_{i}\right)$ and $\mathbf{u}_{1}\left(\boldsymbol{x}-\mathbf{u}_{i}\right)$ are approximated by the weighted mean of three rotated vectors along three axes. After $\left\{\frac{\partial \mathbf{u}_{1}}{\partial \mathbf{u}_{i}}\right\}$ are obtained, we can calculate the above four indices in Eq. (9), Eq. (10) and Eq. (11), and Eq. (12), from the directional derivatives.

\section{Experiments}

Synthetic data experiments. Fig. 4 demonstrated these four distortion indices calculated from idealized tensor fields. The tensors were visualized using superquadric tensor glyphs. Fig. 4 showed that 1 ) the four indices only depend on the orientations, not on the tensor or ODF shape; 2) splay, bend, twist indices provide complementary information about the orientational change, which demonstrate different types of orientational distortions. Note that the twist index for the third tensor field is actually a constant, the index 


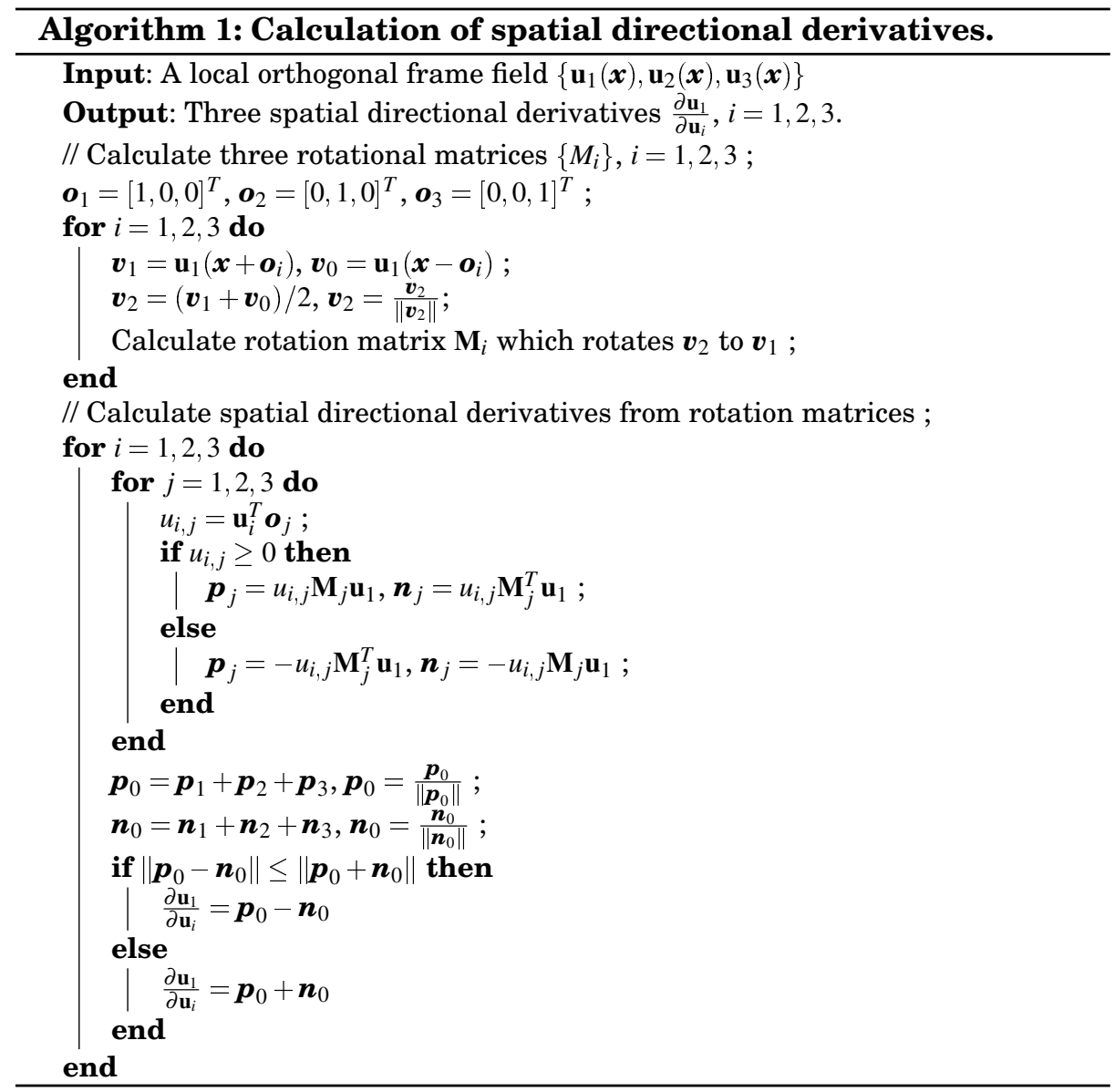

value around the boundary is different due to the Neumann boundary condition used in the calculation. Although the results in Fig. 4 are for tensor fields, the distortion indices are actually determined by the local orthogonal frame field that can be calculated from a general spherical function field.

We compared the four distortion indices with the curving and dispersion indices proposed for tensor fields in [10]. The tensor field in Fig. 5 was used in [10]. It has three areas where the tensors rotate about its three eigenvectors respectively. Fig. 5 shows that 1 ) splay index is similar with dispersion index, and bend index is similar with curving index; 2) the four distortion indices are independent of tensor shapes, while curving and dispersion indices are dependent on tensor shape; 3 ) when the principal directions are well aligned, all distortion indices are close to zero, because they are calculated based on the spatial difference of principal directions. Note that dispersion and curving indices in [10] are only for tensor fields, while the proposed distortion indices work for both tensor field and ODF field. 


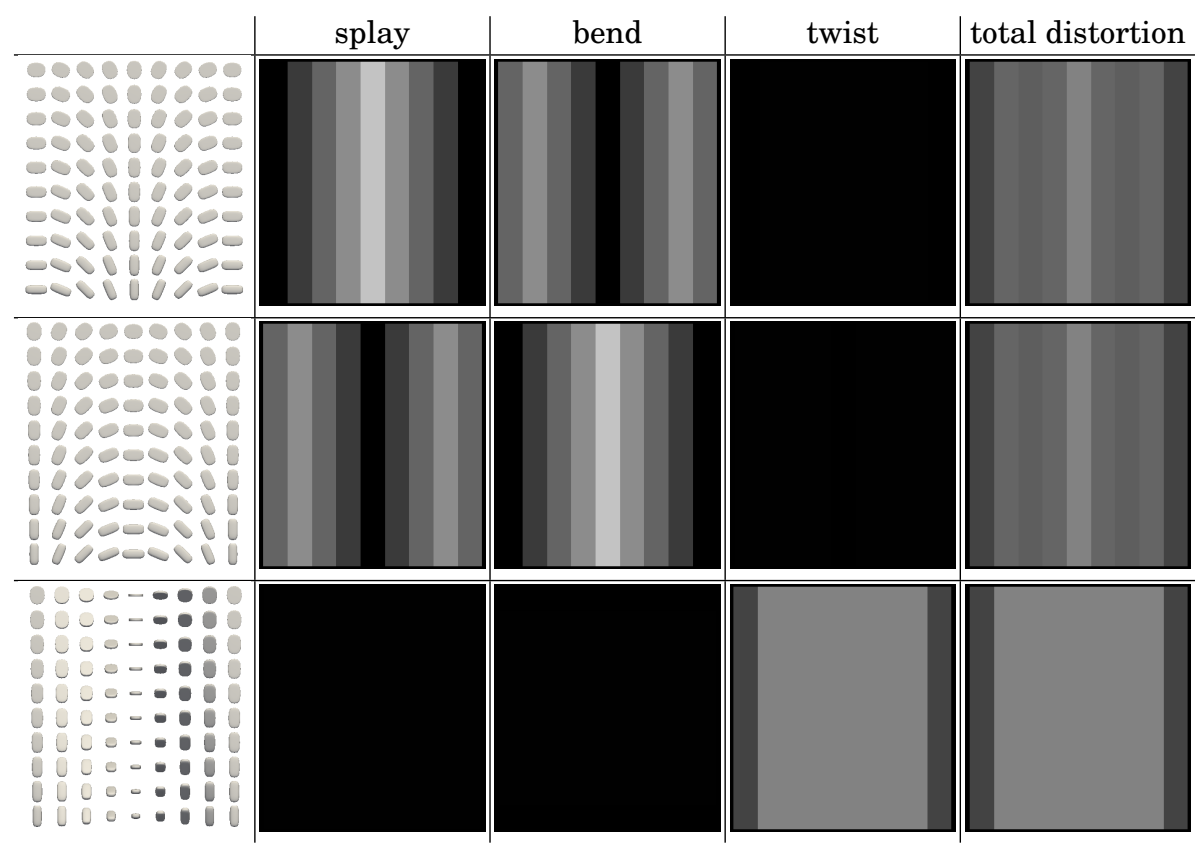

Fig. 4. Distortion indices calculated from different tensor fields.

Real data experiments. The real data is from Human Connectome Project (HCP). It has three shells at $b=1000,2000,3000 \mathrm{~s} / \mathrm{mm}^{2}$, with 90 staggered directions per shell. Non-negative spherical deconvolution (NNSD) [4] was performed to estimate fiber ODFs from three shell DWI data. Peaks were detected from the estimated fODFs with GFA larger than 0.3. OO and OD were calculated from the spherical harmonic representation of fODFs along their principal peaks. Fig. 6 demonstrated OO and OD maps from fODFs, and the total distortion map estimated from the local orthogonal frames of fODFs. The close-up views of fODFs, local orthogonal frames, the six proposed indices for the red region, which is the crossing area of Corpus Callosum and Fornix, were also visualized. The three orientations in the local orthogonal frame in each voxel were visualized using three tubes in red, blue and green color respectively. We performed whole brain streamline tractography on the fODF field using mrtrix ${ }^{3}$. The voxels with GFA larger than 0.3 were used as seed voxels to generate 10000 tracts by using tckgen in mrtrix. The obtained fiber tracts cross the red region were colored by the proposed six scalar indices and visualized using trackvis 4 Fig. 6 showed that 1) OO is high in anisotropic areas with well-aligned directions, while OD is high in isotropic or crossing areas. 2) the four distortion indices

\footnotetext{
$3^{3}$ http://www.mrtrix.org/

${ }^{4}$ http://trackvis.org
} 


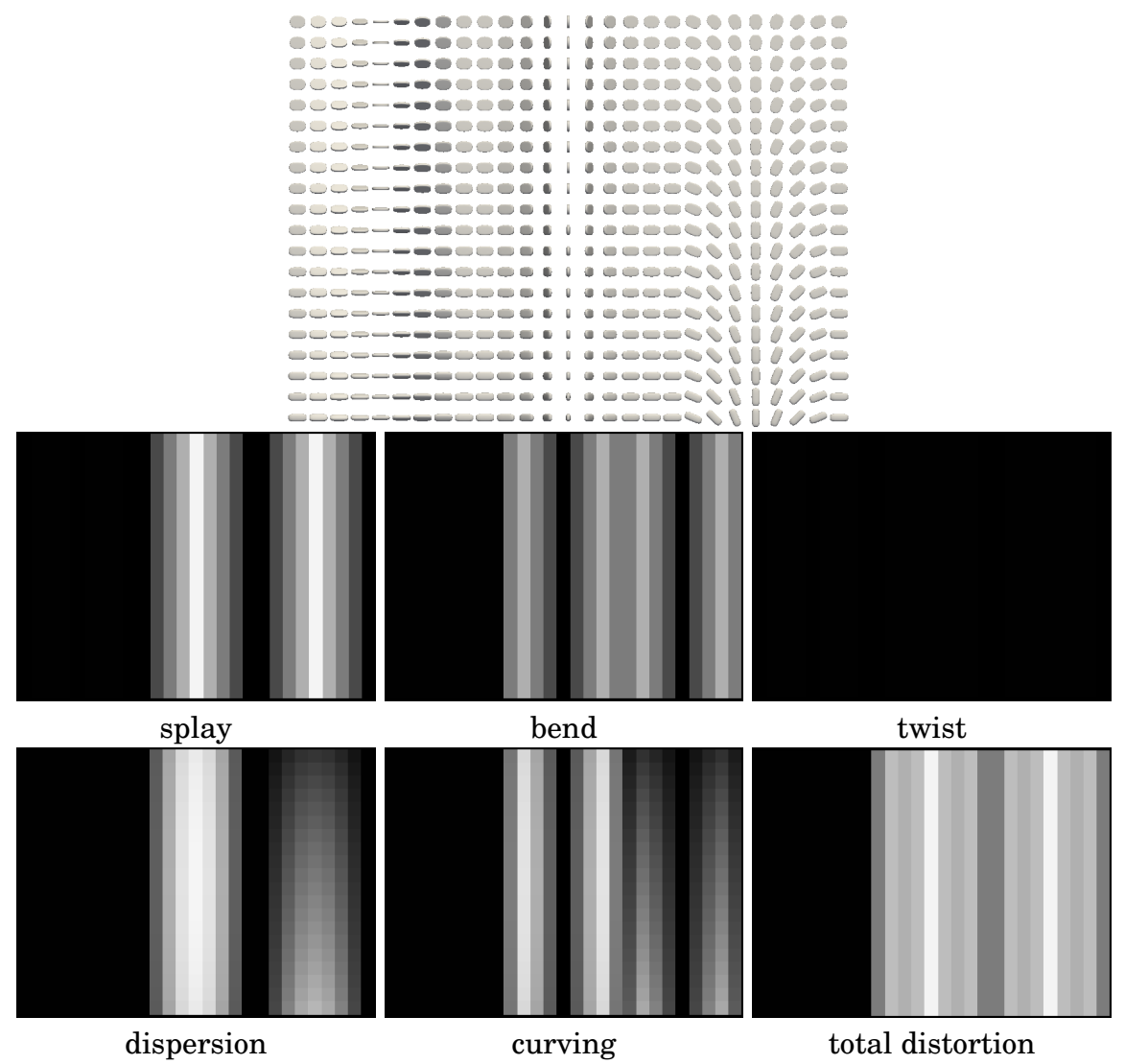

Fig. 5. Dispersion, curving [10], and the proposed four distortion indices calculated from a tensor field.

are low in areas with well aligned principal directions, and zero in isotropic voxels without peaks. 3 ) total distortion index is high in areas with highly curved fibers or crossing fibers. 4) Although splay, bend, twist indices may be separable (e.g., one is large while another one is close to zero) in synthetic data, in real data, these three types of distortions normally occur together, especially for bending and splaying.

\section{Conclusion}

In this paper, we proposed a unified mathematical framework called Director Field Analysis (DFA) to analyze a spherical function field and its extracted peaks. First, we define the orientational order (OD) and the orientational dispersion (OD) indices in voxels. OD is more general and natural than previous dispersion index proposed in NODDI [14]. Second, we define a local orthogonal frame in each anisotropic voxel with the principal 
peak as its first axis, and the other two axes describing the local spatial change directions of principal peaks. Third, from the extracted local orthogonal frames in voxels, DFA estimates three distortion indices (splay, bend, twist) which is able to distinguish three types of distortions, and a total distortion index. To our knowledge, it is the first work to quantitatively describe orientational distortion (splay, twist, and bend) in diffusion MRI data. Considering the proposed scalar indices are sensitive to different distortions of principal directions, these indices have potential in voxel-based analysis and tract-based analysis for group studies.

\section{References}

1. Denis Andrienko. Introduction to liquid crystals. Bad Marienberg, 2006.

2. Peter J Basser. New histological and physiological stains derived from diffusiontensor mr images. Annals of the New York Academy of Sciences, 123-138, 1997.

3. Peter J. Basser, James Mattiello, and Denis LeBihan. MR diffusion tensor spectroscropy and imaging. Biophysical Journal, 66:259-267, 1994.

4. Jian Cheng, Rachid Deriche, Tianzi Jiang, Dinggang Shen, and Pew-Thian Yap. Non-Negative Spherical Deconvolution (NNSD) for estimation of fiber Orientation Distribution Function in single-/multi-shell diffusion MRI. NeuroImage, 101:750-764, 2014.

5. G Kindlmann, D B Ennis, R T Whitaker, and C-F Westin. Diffusion Tensor Analysis with Invariant Gradients and Rotation Tangents. IEEE Transactions on Medical Imaging, 26(11):1483-1499, 2007.

6. Christian Lessig, Tyler de Witt, and Eugene Fiume. Efficient and accurate rotation of finite spherical harmonics expansions. Journal of Computational Physics, 231(2):243-250, 2012.

7. Sinisa Pajevic, Akram Aldroubi, and Peter J Basser. A continuous tensor field approximation of discrete dt-mri data for extracting microstructural and architectural features of tissue. Journal of magnetic resonance, 154(1):85-100, 2002.

8. C. Pierpaoli and P. Basser. Toward a Quantitative Assessment of Diffusion Anisotropy. Magnetic Resonance in Medicine, 36:893-906, 1996.

9. Emmanuel Piuze, Jon Sporring, and Kaleem Siddiqi. Maurer-Cartan forms for fields on surfaces: application to heart fiber geometry. IEEE transactions on pattern analysis and machine intelligence, 37(12):2492-2504, 2015.

10. P Savadjiev, GL Kindlmann, S Bouix, ME Shenton, and C-F Westin. Local white matter geometry from diffusion tensor gradients. NeuroImage, 2010.

11. Filip Szczepankiewicz, Samo Lasič, Danielle van Westen, Pia C Sundgren, Elisabet Englund, Carl-Fredrik Westin, Freddy Ståhlberg, Jimmy Lätt, Daniel Topgaard, and Markus Nilsson. Quantification of microscopic diffusion anisotropy disentangles effects of orientation dispersion from microstructure: applications in healthy volunteers and in brain tumors. NeuroImage, 104:241-252, 2015.

12. Peter Savadjiev, Steven W Zucker, and Kaleem Siddiqi. On the differential geometry of $3 \mathrm{~d}$ flow patterns: Generalized helicoids and diffusion mri analysis. In ICCV , 1-8, 2007.

13. David S. Tuch. Q-ball imaging. Magnetic Resonance in Medicine, 2004.

14. Hui Zhang, Torben Schneider, Claudia A Wheeler-Kingshott, and Daniel C Alexander. NODDI: practical in vivo neurite orientation dispersion and density imaging of the human brain. Neuroimage, 61(4):1000-1016, 2012. 


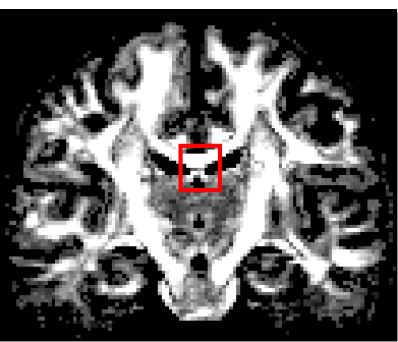

OO

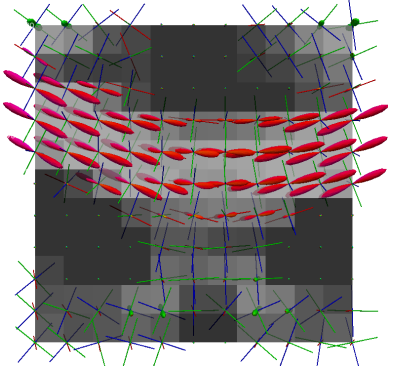

$\mathrm{OO}$

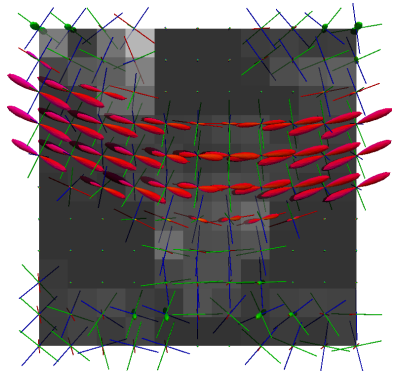

splay

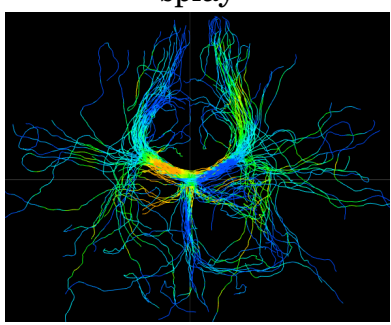

$\mathrm{OO}$

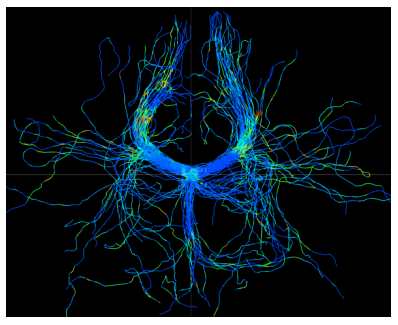

splay

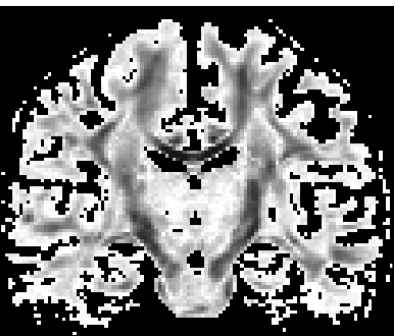

OD

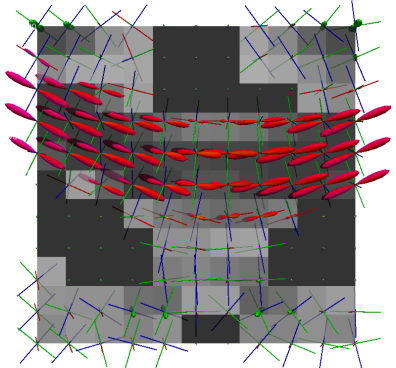

OD

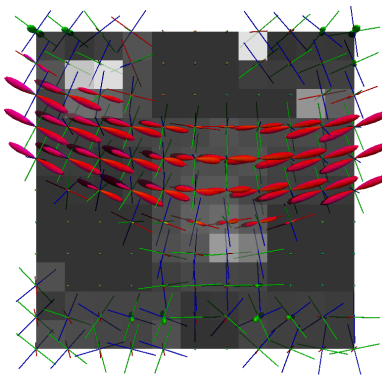

bend

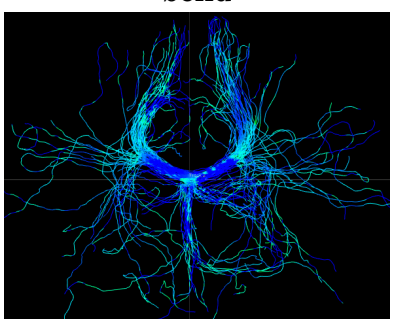

OD

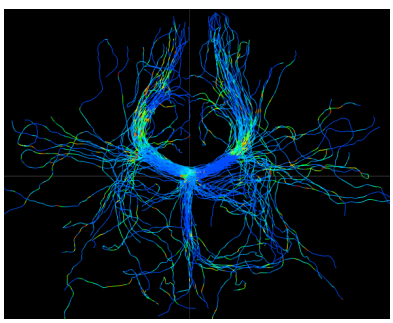

bend

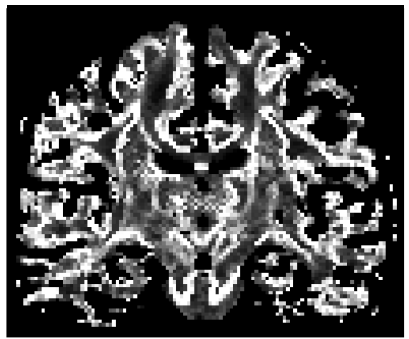

total distortion

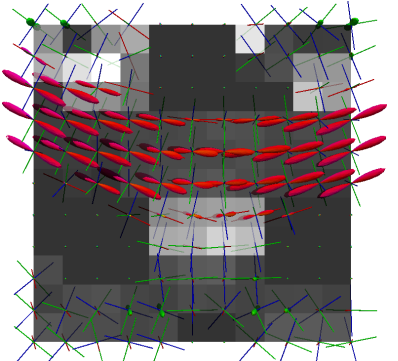

total distortion

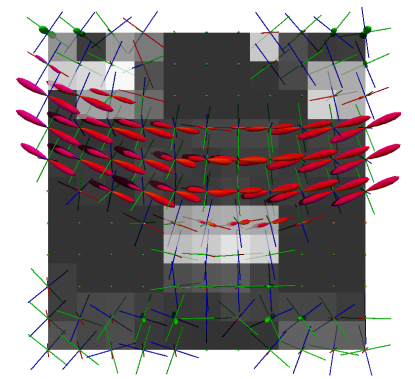

twist

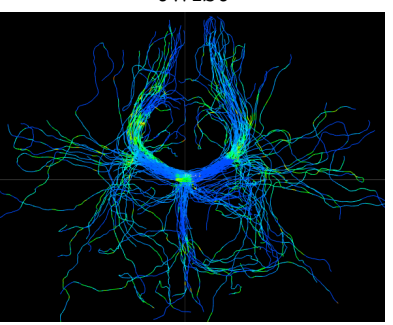

total distortion

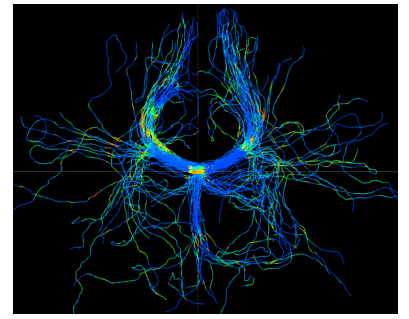

twist

Fig. 6. First row: OO, OD and total distortion maps calculated from fODFs in multi-shell HCP data. 2-3 rows: the close-up views of fODFs, local orthogonal frames, the six proposed indices in the red region. 4-5 rows: fiber tracts cross the red region were colored by six indices. 